\title{
Armonización contable en España con las directivas de la CEE
}

\author{
LEANDRO CAÑIBANO CALVO*
}

\section{De la normalización \\ a la armonización contable}

【 na de las notas más características de las sociedades económicamente más avanzadas, es la de su mayor transparencia informativa; sus agentes económicos se ven impelidos a comunicar periódicamente una información precisa sobre su situación financiera y sobre los resultados alcanzados con sus operaciones. Esta obligación se deriva tanto de las normas legales existentes, como de los hábitos fuertemente enraizados en la comunidad económica internacional, que exige, de día en día, más y más información financiera a las empresas de negocios, delineando al propio tiempo los criterios y pautas que deben servir de base para lograr que la misma sea objetiva y fiable.

* Catedrático de Economía Financiera y Contabilidad de la Universidad Autónoma de Madrid.

Vicepresidente 1o. de la Asociación Espainola de Contabilidad y Administración de Empresas.
Puesto que la información financiera que las empresas han de hacer pública periódicamente, se obtiene a partir de una base de datos contables, no resulta extraño en modo alguno que la forma de hacer objetiva y fiable la mencionada información se haya abordado a partir de la normalización contable, esto es, de elevar al rango de norma el conjunto de principios, criterios y procedimientos que, en un determinado contexto, deben ser utilizados para preparar la información contable-financiera, en especial, la que forma parte de los estados financieros que han de ser hechos públicos con carácter periódico.

La dimensión internacional del mundo de los negocios, el establecimiento de espacios económicos más amplios que los delimitados por las propias fronteras nacionales, ha llevado la normalización 


\title{
El facsímil
}

\author{
ORIGINAL DE LA CARTA DE COLON \\ (PRIMIRA PAGINA)
}

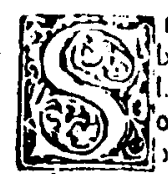

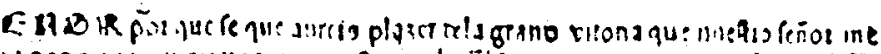

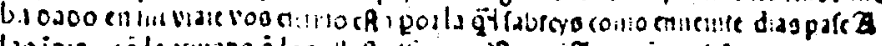

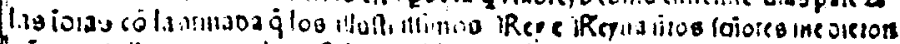

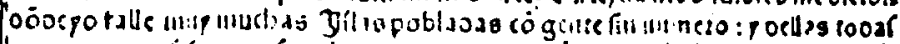

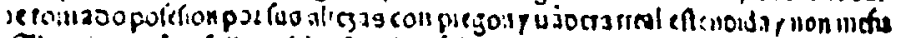

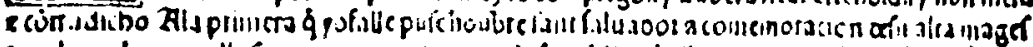

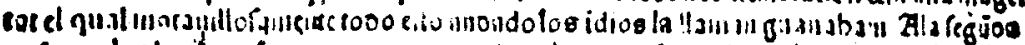

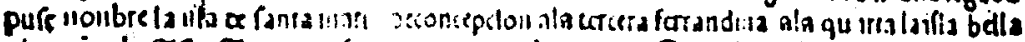

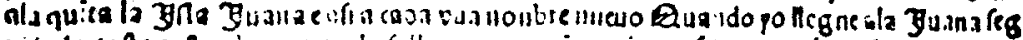

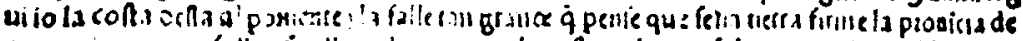

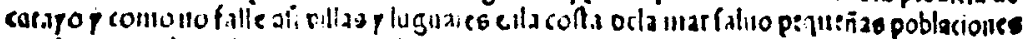

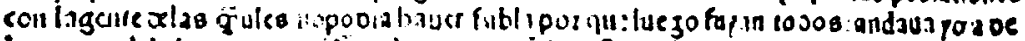

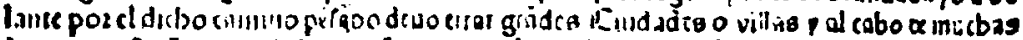

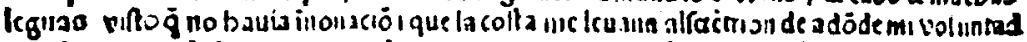

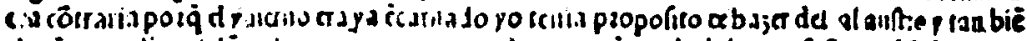

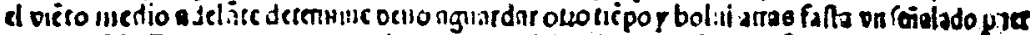

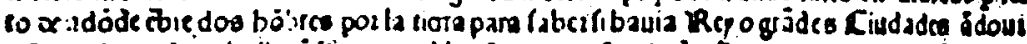

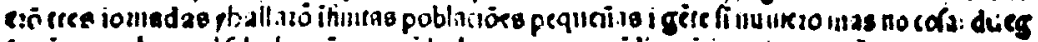

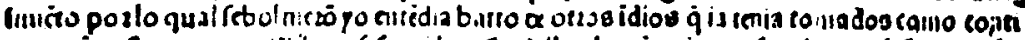

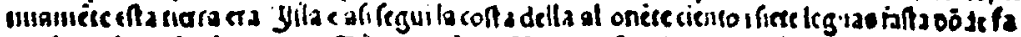

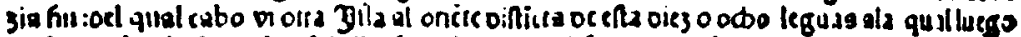

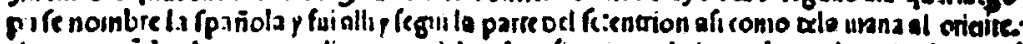

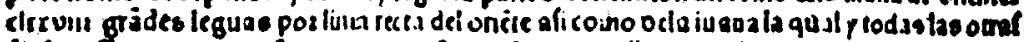

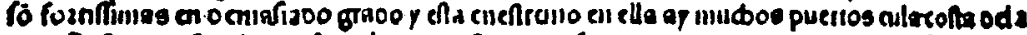

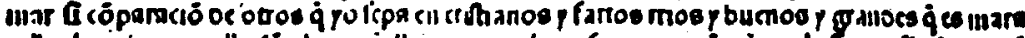

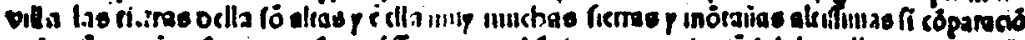

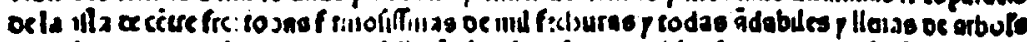

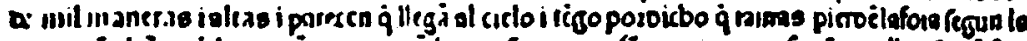

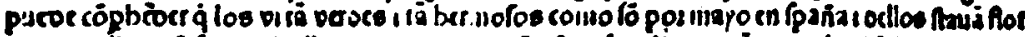

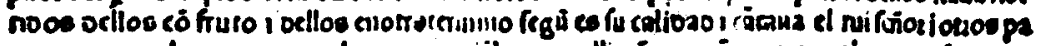

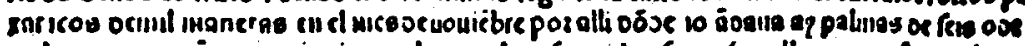
oc bo mancras ḡ es aoniracion vilae poz la oufounideo fennole oellas inas aficono lose

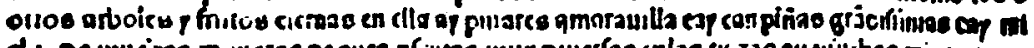

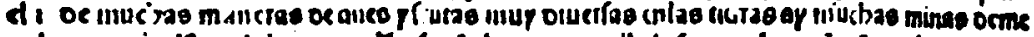

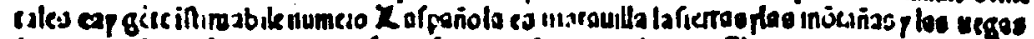

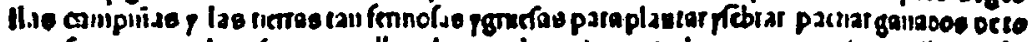
oas fuctiz para broiknon oc vilise eligares los pacros ocla mar aqui liu bauria donas fiu

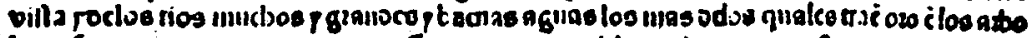

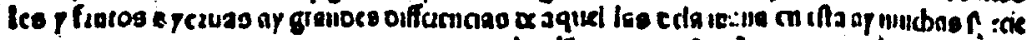

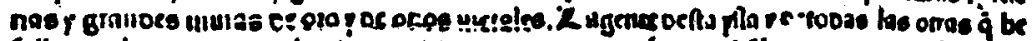
fnilaou y baaloo:ntapa banibo noticia anoen tooos od́lioas böbre pungexce oft coino fre unavies los pare baun que alginas nugoce fe cobriau vu iolo lugaz có una foia oe pre

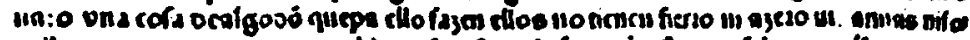

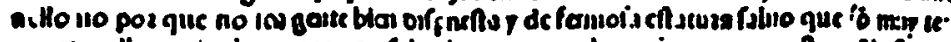

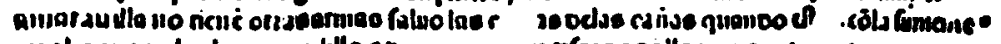

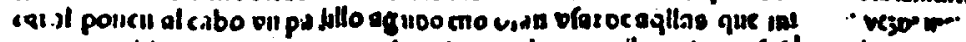

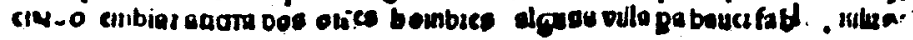


contable más allá de los ámbitos en que inicialmente surgió. Los límites nacionales dejan de tener sentido cuando nos encontramos ante una perspectiva internacional, razón por la cual del camino de la normalización se ha pasado al de la armonización contable, esto es, de una norma para cada país a una norma que sirva de base a un conjunto cada vez más amplio de países. Este es el caso de la Comunidad Económica Europea (CEE), cuyos Estados Miembros habían recorrido, cada uno según su propia idiosincrasia, instituciones, hábitos culturales, etc., un cierto camino en pro de la normalización contable, habiendo sido preciso encajar cada una de estas iniciativas dentro de un planteamiento más general, con objeto de que finalmente pueda llegarse al establecimiento de un auténtico mercado común.

La creación de un mercado común exige la eliminación de las barreras existentes para dar fluidez a las transacciones intracomunitarias, eliminando los obstáculos que pudieran oponerse a la libertad de establecimiento y a la creación de un entorno común para las sociedades de toda la Comunidad. El artículo 54.3.G. del Tratado de Roma, autoriza al Consejo y a la Comisión a efectuar una coordinación, en la medida necesaria, para hacer equivalentes las garantías que son exigidas a las sociedades por los Estados Miembros, para proteger los intereses tanto de los socios como de terceros.

Los esfuerzos de la CEE para armonizar las normas contables forman parte del programa de armonización del derecho de sociedades, habiendo sido acometido éste a través de la promulgación de diversas Directivas. (1)

\section{Directivas de la CEE}

Dentro del marco del derecho de sociedades, han sido adoptadas tres Directivas de índole contable hasta la fecha presente:

La 4a Directiva de 25 de julio de 1978 sobre "Cuentas Anuales", la 7a Directiva de 13 de julio de 1983 sobre "Consolidación de Cuentas" y la 8a Directiva de 10 de abril de 1984 sobre la "Habilitación legal de las personas responsables de llevar a cabo la auditoría de los documentos contables".

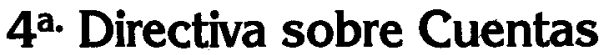 Anuales}

Casi 10 años fueron necesarios para concluir las discusiones previas a la promulgación de la 4a Directiva. La duración de estas negociaciones no fue originada por un desacuerdo sobre las necesidades de armonizar las normas contables vigentes en los diferentes países, fue fruto de las profundas diferencias existentes en muchos casos en las prácticas contables establecidas en los diferentes Estados Miembros. Tales diferencias se extendían también a los objetivos asignados a la información financiera, ya que mientras que para unos su orientación debía ser el mercado bursátil, otros la consideraban más vinculada al ámbito fiscal, mientras que algunos otros pretendían que la misma

(1) Una Directiva es un instrumento legal que contiene obligaciones de carácter mínimo dirigido a los Estados Miembros, los cuales deben recoger, dentro de su normativa legal, todas las disposiciones que crean convenientes para obligar, dentro de su ámbito, al cumplimiento, como míninho, de los preceptos contenidos en la mencionada Directiva. 
debía responder a objetivos macroeconómicos.

Por fin, en la fecha anteriormente indicada, la Directiva vio la luz, dirigiéndose la misma a las sociedades de capitales que limitan su responsabilidad, estableciendo los requerimientos mínimos legales respecto a la información financiera que éstas debían poner a disposición del público. En concreto, contiene normas sobre la presentación y valoración de las cuentas anuales, sobre la publicidad que debe darse a las mismas y sobre la obligación de someter éstas a la auditoría realizada por un experto independiente.

Las cuentas anuales integradas por el balance, la cuenta de pérdidas y ganancias y el anexo o notas complementarias, deben mostrar una imagen fiel del patrimonio, de la situación financiera y de los resultados. Cuando las normas contenidas en la Directiva fueran insuficientes para ofrecer dicha imagen fiel, deberán ofrecer en el anexo las correspondientes indicaciones, con objeto de llegar a mostrar la citada imagen fiel.

Igualmente, cuando la aplicación de una norma contenida en la Directiva se revele como incompatible con el objetivo de ofrecer una imagen fiel, dicha disposición puede dejar de ser cumplida, siempre y cuando se trate de un caso excepcional, poniendo de manifiesto en el anexo tanto las normas que han dejado de cumplirse, como los criterios que han sido aplicados en su lugar y la incidencia que sobre el patrimonio, la situación financiera y los resultados tienen los criterios contables que finalmente han sido utilizados.
El objetivo de imagen fiel, tal como ha quedado expresado por la Directiva, ha dado lugar a ríos de tinta en la literatura contable, sobre todo en aquellos países en que dicho principio resultaba extraño. Ciertamente, la facultad que confiere al profesional de poder derogar una norma y aplicar la que él considere más relevante para ofrecer la deseada imagen fiel, choca con las habituales prácticas seguidas en una buena porción de países europeos, que han venido poniendo siempre por delante a la norma. Lo cierto del caso es que, incluso en los países donde mayor tradición tiene esta forma de hacer, raramente los profesionales se apartan de la cobertura de las normas, porque en otro caso, toda la carga de la prueba recae sobre quienes toman dicha opción. Por el momento, pensamos que la trascendencia de este objetivo de imagen fiel radica más en la propia concepción filosófica del mismo, que en la importancia que en la práctica, hasta este mòmento, está teniendo.

Las anteriores cuentas anuales deben presentarse, en términos comparativos, esto es, junto a las cifras del ejercicio corriente deben ofrecerse las del ejercicio precedente y de acuerdo con los modelos contenidos en la propia Directiva (2), valorándose las diferentes partidas de acuerdo con los criterios de valoración generales y específicos contenidos en la mencionada Directiva.

Tales criterios valorativos incluyen, de una parte, los conocidos principios contables básicos de: "empresa en fun-

(2) En nuestra obra "Contabilidad: Análisis Contable de la Realidad Económica", Pirámide, Madrid 1987, Cap. 19 , se efectúa una presentación esquemática de dichos modelos. 
cionamiento", "uniformidad", "prudencia valorativa", "correlación de ingresos y gastos", "devengo" y "no compensación", así como también el principio contable del coste histórico. Las reglas específicas se refieren a partidas tales como los gastos de constitución y primer establecimiento, los costes de investigación y desarrollo, el fondo de comercio, los activos fijos, los activos circulantes, las existencias, la constitución de provisiones para riesgos y gastos, etc.

$\mathrm{El}$ anexo constituye un documento informativo de primera magnitud, ya que en él deben incluirse notas relativas a los principios contables y métodos de valoración aplicados, a las empresas filiales y participadas, a la estructura del capital, obligaciones garantizadas a largo plazo, compromisos financieros no incluidos en el balance, volúmenes de ventas por segmentos industriales y geográficos, número promedio de personas empleadas y sus diferentes categorías, impuestos anticipados o diferidos, relaciones económicas entre la sociedad y los miembros de su consejo de administración. A esta lista deben ser añadidas, en no pocos casos, informaciones complementarias exigidas a lo largo de diferentes disposiciones de la Directiva siempre y cuando se den los supuestos en ellas contenidos.

Independientemente de los anteriores documentos de naturaleza contable, la Directiva exige también la presentación de un informe anual de gestión a través del cual se debe ofrecer una visión correcta de la evolución de los negocios de la sociedad y de la situación de la misma, debiendo ponerse también de manifiesto cualquier acontecimiento im- portante que ocurra después del cierre del balance, así como la evolución previsible de la empresa, las actividades que la misma realiza en el campo de la investigación y desarrollo y la información concerniente a la adquisición de las acciones propias.

Todas las sociedades deben someter sus cuentas anuales a auditoría, que habrá de realizar un profesional legalmente habilitado por el Estado correspondiente, quedando exceptuadas solamente las pequeñas empresas, entendiendo por tales aquellas que no superen dos de los tres límites siguientes: balance total 1,55 millones de ECUS (1,77 millones de dólares), cifra de negocios 3,2 millones de ECUS (3,65 millones de dólares), número medio de empleados 50 .

Las cuentas anuales aprobadas por la Junta de Accionistas, el informe de gestión y el informe de los auditores deben ser hechos públicos a través de los procedimientos establecidos en la Primera Directiva, consistentes en la inclusión de tales documentos en un archivo abierto dentro de un registro central y su publicación total o resumida en un diario de difusión nacional. Existen, naturalmente, excepciones para las pequeñas y medianas empresas.

Con la excepción de Italia entre los miembros de la CEE más antiguos y Portugal, Grecia y España entre los más modernos, todos los restantes han adaptado sus legislaciones respectivas al contenido de la 4a Directiva anteriormente comentada. Los pasos dados a este respecto por España y Portugal hacen prever que, en el próximo año 1989, la legislación de ambos países 


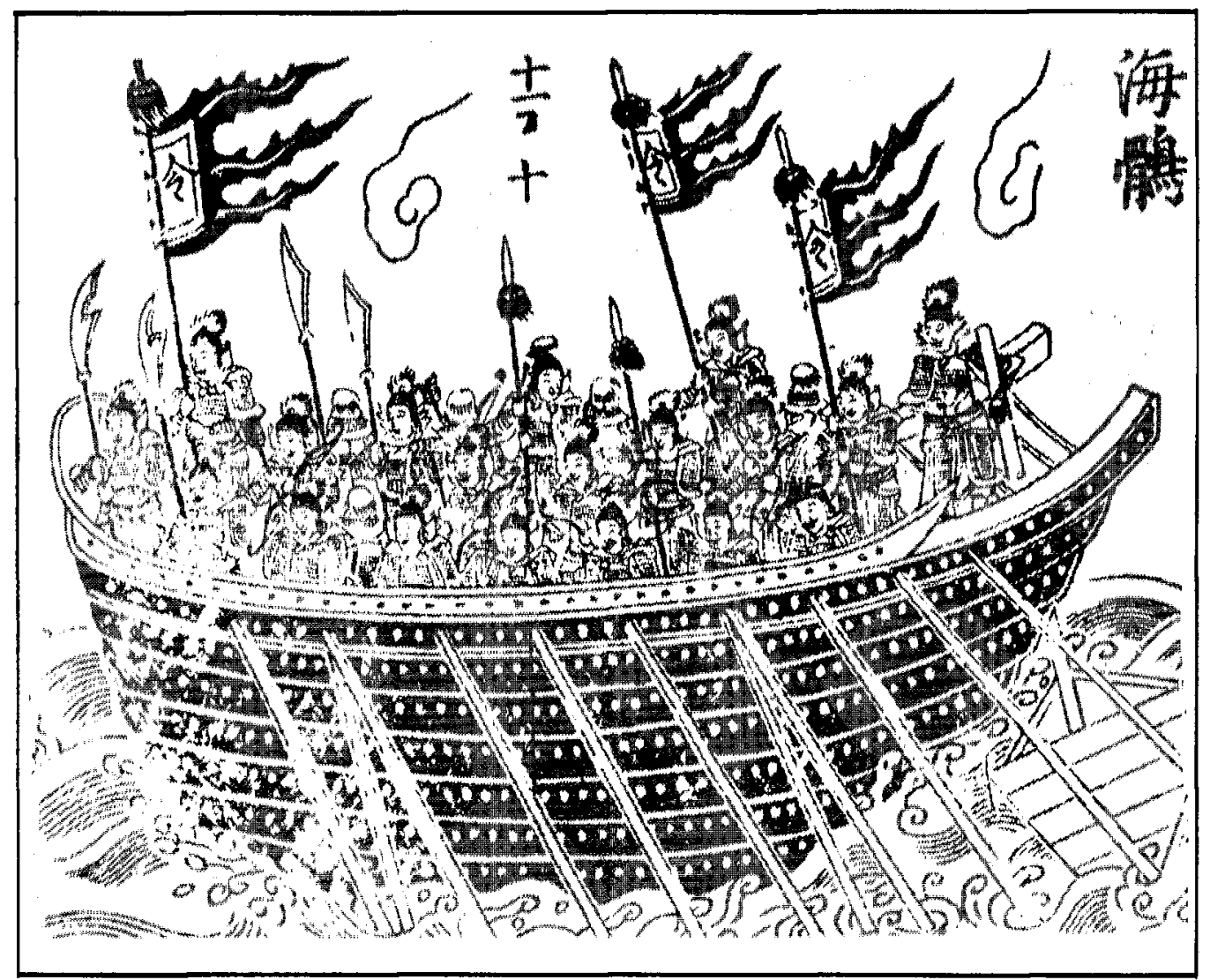

quede armonizada con las Directivas comunitarias comentadas en este trabajo.

\section{7a. Directiva sobre Cuentas Consolidadas}

El proceso de gestación de esta Directiva fue también bastante largo, ya que se necesitaron casi 7 años para que la misma fuese finalmente aprobada. Las principales razones que explican la larga duración de este proceso son, de una parte, la poca familiaridad que la mayoría de los Estados Miembros tenían con las técnicas de consolidación, y, de otra, el hecho de que existieran dos concepciones distintas sobre la forma de determinar el conjunto de la consolidación, basada una en el criterio económico de unidad de dirección, mientras que se apoyaba la otra, en el concepto de poder legal de control. Este último fue el que finalmente prevaleció, pero no de una forma total y absoluta, ya que fueron necesarias también algunas concesiones adicionales.

La consolidación resulta obligada cuando una sociedad tiene el poder legal de control de otra u otras, el cual se da siempre y cuando se cumplan algunos de los siguientes puntos: tenga una mayoría de votos, o el derecho de colocar o quitar la mayoría de los miembros del Consejo, o el derecho de ejercer una influencia dominante de acuerdo con un contrato, o el control de una mayoría de los votos legales en base a un acuerdo 
de los accionistas.

Los Estados Miembros pueden exigir también la presentación de cuentas consolidadas cuando existe un control de "facto" dándose el mismo cuando la sociedad matriz haya situado una mayoría de los miembros del Consejo durante dos años economicos sucesivos, o ejerza una influencia dominante, o dirija por sí misma una firma subsidiaria sobre bases unificadas.

No obstante lo anterior, existen diversas posibilidades de excepción de la obligación de consolidar, como por ejemplo, cuando la sociedad matriz no tenga el carácter de sociedad de capitales que limite su responsabilidad. Los holdings financieros también pueden ser excepcionados de esta obligación así como los grupos calificados como pequeños salvo que incluyan una sociedad cotizada en bolsa.

Además una empresa puede ser excluida de las cuentas consolidadas del grupo cuando su inclusión resulte inconsistente con respecto al objetivo de imagen fiel, existan restricciones a largo plazo que entorpezcan el control por parte de la empresa matriz, la información necesaria no pueda ser obtenida sin realizar gastos desproporcionados o retrasos excesivos, las acciones de la empresa se mantengan con vistas a una venta posterior o las actividades sean tan diferentes que su

(3) Se entiende por grupos pequeños los que no rebasan dos de los tres límites siguientes: Balance total 6,2 millones de ECUS (7,07 millones de dólares), cifra de negocios 12,8 millones de ECUS (14,60 millones de dólares), número medio de empleados 250. Los límites anteriores expresados en ECUS pueden ser multiplieados por 2,5 durante un periodo de transición de 10 affos durante el cual también el número de empleados podría considerarse hasta un máximo de 500 . inclusión pudiera ser incompatible con el mencionado objetivo de imagen fiel.

Para la elaboración de las cuentas consolidadas habrá de seguirse algunos de los métodos de consolidación expuestos por la Directiva, la cual prevé tres a este respecto: integración global cuando las participaciones sean superiores al $50 \%$, puesta en equivalencia para los casos en que éstas se encuentren situadas entre el 20 y el 50\%, e integración proporcional cuando se trate de empresas comunes o sistemas de intereses afines. Las cuentas consolidades deberán mostrar los activos, pasivos, posición financiera y perdidas y ganancias de las sociedades incluidas en la consolidación, lo cual requerirá la desaparición de créditos y débitos mutuos, de ingresos y gastos dentro del grupo y de las pérdidas y ganancias originadas por operaciones entre dichas empresas. En el anexo deberán mencionarse, además de cuestiones similares a las anteriormente señaladas por la 4! Directiva, otras más específicas de las cuentas consolidadas como son: información sobre compañías incluidas o excluidas de la consolidación, empresas asociadas consolidadas proporcionalmente, etc.

Las anteriores cuentas consolidadas deben ser sometidas a auditoría y hechas públicas en términos similares a los indicados con anterioridad. Igualmente, se preparará un informe de gestión consolidado con contenido similar al anteriormente indicado.

Los Estados Miembros han de armonizar su legislación con esta Directiva antes del 12 de enero de 1988, debiendo comenzar a aplicarse la misma en el 
ejercicio económico 1990. La mayor parte de los países comunitarios han aprovechado diversos cambios legislativos para incorporar a sus disposiciones mercantiles el contenido de la Directiva comentada; ésto ha ocurrido con Francia en 1985, Alemania en el mismo año, Holanda, etc.

\section{$8^{a}$ Directiva sobre hablitación de los Auditores}

La Directiva 8" no se refiere al reconocimiento mutuo de grados, diplomas $u$ otras cualificaciones requeridas por los Estados Miembros para la realización de las auditorías legales. Tampoco trata sobre el efectivo ejercicio de la libertad de ofrecer servicios o sobre la libertad de establecimiento de una persona autorizada en un Estado Miembro para practicar en otro Estado Miembro. Estos problemas serán solventados dentro de otro contexto. La Directiva, sin embargo, permite a los Estados Miembros que autoricen a aquellas personas que hayan obtenido todas o parte de sus cualificaciones en otro Estado si dichos Estados consideran tales cualificaciones como equivalentes a las requeridas por su ley nacional.

La habilitación de una persona física requiere la superación de un examen final, organizado o reconocido por el Estado, que versará sobre un conjunto de materias de naturaleza jurídica, económica y contable que la propia Directiva señala. Cuando algún título profesional o universitario, para su expedición, requiera haber superado todas 0 algunas de las anteriores materias, el Estado correspondiente podrá dispensar de la realización de dicho examen total o parcialmente. Además se exigirá una experiencia práctica durante al menos tres años, realizada bajo la dirección de personas que tengan adecuadas garantías. Los Estados Miembros podrán también instrumentar el acceso a la condición de auditor de personas con 15 años de experiencia relevante o con una combinación de 7 años de experiencia y prácticas en el sentido anteriormente expuesto; en ambos casos dichas personas habrán de aprobar también el examen de competencia profesional al que anteriormente hemos hecho referencia.

Los Estados Miembros podrán autorizar la existencia de firmas de auditores siempre y cuando que éstas satisfagan ciertas condiciones, tales como: que la auditoría sea realizada en nombre de la firma por un auditor legalmente habilitado, que una mayoría de los derechos de voto sea sostenido por auditores legalmente habilitados, que una mayoría de los miembros de la dirección de la firma sean auditores igualmente habilitados.

La habilitación podrá ser otorgada tan sólo a personas de buena reputación que no lleven a cabo ninguna actividad incompatible según la ley del Estado Miembro correspondiente. El requisito de independencia se considera como absolutamente esencial, sin embargo la Directiva no incluye reglas específicas sobre cómo poder apreciar la misma.

La implantación de esta Directiva corre pareja, en cuanto a fechas se refiere, con las anteriormente indicadas para las cuentas consolidadas, por lo tanto nos remitimos a lo ya dicho. 


\section{Otros desarrollos}

Además de las anteriores Directivas que constituyen el armazón fundamental de las disposiciones de índole contable en el ámbito comunitario, debemos señalar que también existe otra de promulgación más reciente, en concreto el 8 de diciembre de 1986, relativa a las Cuentas Anuales y a las Cuentas Consolidadas de los Bancos y otras entidades financieras. Dicha Directiva era necesaria al haber sido expresamente excluidas de las Directivas precedentes este tipo de entidades. Su implantación en la legislación de los diferentes países deberá producirse antes del 31 de diciembre de 1990 y su aplicación a las cuentas deberá tener lugar en el ejercicio que comienza el 1은 de enero de 1993.

Un proceso similar al seguido para los bancos ha comenzado ya para las compañías aseguradoras que cuentan desde 1986 con una propuesta de Directiva relativa a sus cuentas anuales y cuentas consolidadas.

También existen propuestas de ampliación de las Directivas comentadas especialmente en lo que se refiere a su ámbito de aplicación, que se trata de hacer extensivo a otro género de entidades, tales como las cooperativas y otros tipos de asociaciones e, igualmente, a las sucursales abiertas en un Estado Miembro por sociedades procedentes de otro Estado.

\section{Principios y normas de contabilidad en España}

Durante largo tiempo las empresas españolas han venido considerando la rendición de cuentas anuales como un requisito más de índole fiscal, dada la preeminencia de este usuario de la información contable-financiera sobre el resto de los posibles destinatarios de la misma. Durante la década de los 70 empieza a notarse un cierto cambio, especialmente por la relevancia que adquieren otros importantes usuarios de las cuentas anuales de las empresas, como son sus propios empleados, los inversores potenciales, los analistas económico-financieros profesionales, las entidades crediticias, especialmente las de naturaleza internacional, etc. ${ }^{(4)}$.

La ausencia de normas contables dio pie a que la normativa fiscal se adentrara en este campo, dejando secuelas que aún hoy perduran, impidiendo, en no pocas ocasiones, que las cuentas anuales constituyan una auténtica imagen fiel de la situación financiera y de los resultados alcanzados por las empresas.

Con la promulgación del Plan General de Contabilidad en 1973 se dio un primer e importante paso en pro de la normalización de la contabilidad y de las cuentas anuales a presentar a terceros. Las sucesivas adaptaciones sectoriales que a partir de la fecha antes citada han ido promulgándose, así como otras diversas normas que cubren campos tales como la consolidación de las cuentas de los grupos de sociedades, la contabilidad analítica, la contabilización del IVA, o el Plan General de Contabilidad, para la pequeña y mediana empresa, suponen

(4) Una visión más detallada de la evolución aludida puede verse en nuestro trabajo "Cambios en la información financiera de la empresa española". En: XXV Años de Contabllidad Universitarla en Espafia. - Instituto de Planificación Contable, Madrid 1988, pp. 86-130. 


\section{$-=\equiv 7 \times 6742$}

Hindú (s. III a. de J.C.)

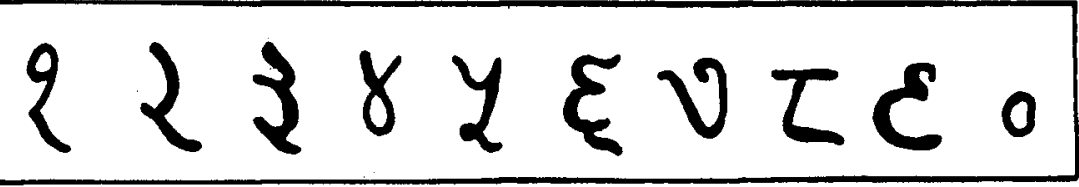

Hindis (s. $X X$ d. de J.C.)

\section{$127 \leq 96789$}

Arabe Occidental (s. X)

\section{I ${ }^{\prime} \mu \kappa^{5} 4 \vee \wedge 9$.}

Arabe Oriental (s. h)

\section{0}

Nacimiento de nuestro sistema de numeración.

una buena muestra del desarrollo alcanzado por la normalización contable desde el plano oficial. Esto cobra aún más importancia si tenemos en cuenta lo lejanas que en esas fechas quedaban nuestras disposiciones mercantiles, Código de Comercio y Ley de Sociedades Anónimas-, de las de igual naturaleza existentes en los países europeos.

No obstante, pese al ingente esfuerzo realizado en este aspecto por el Instituto de Planificación Contable (5), las necesidades sentidas por los profesionales a este respecto iban mucho más allá, especialmente cuando tales profesionales desempeñaban su tarea como auditores independientes de cuentas, toda vez que habían de comprometer su opinión con la representatividad de las cuentas de una

(5) Actualmente sus funciones se encuentran incorporadas al Instituto de Contabilidad y Auditoría de Cuentas creado por la Ley de Auditoría de Cuentas de 12 de julio de 1988. 
y otra compañía, de acuerdo con principios de contabilidad generalmente aceptados, muchos de los cuales no quedaban respaldados por pronunciamientos autóctonos, sino por declaraciones foráneas procedentes de organizaciones de gran prestigio en el contexto internacional, pero al fin y al cabo ajenas a nuestro entorno cotidiano. Esta situación dio pie a la creación de la Asociación Española de Contabilidad y Administración de Empresas (AECA) que desde su inicio se comprometió en la tarea de emitir unos principios y normas de contabilidad que llegaran a alcanzar en nuestro país suficiente respaldo competente, de manera tal que les fuera otorgada por los profesionales, por sus corporaciones representativas, la condición de generalmente aceptados.

Desde su fundación en 1979, la Comisión de Principios y Normas de Contabilidad de AECA ha venido emitiendo pronunciamientos sobre las partidas más significativas de los estados financieros, cubriendo los siguientes campos: "principios contables básicos", "inmovilizado material", "inmovilizado inmaterial y gastos amortizables", "diferencias de cambio en moneda extranjera", "proveedores, acreedores y otras cuentas a pagar", "clientes, deudores y otras cuentas a cobrar", "ajustes por periodificación y cobros y pagos diferidos", "existencias", "impuesto sobre beneficios", "recursos propios", "provisiones, contingencias y acontecimientos posteriores al cierre de los estados financieros", "ingresos" e "ingresos diferidos". En fecha inmediata serán emitidos dos nuevos pronunciamientos sobre "inversiones financieras", "fondo de reversión" y "fondos de pensiones". El ritmo seguido en la emisión de los anteriores documentos hace prever que antes del comienzo de 1990 se contará con un importante conjunto de ellos que otorguen cobertura a las partidas más representativas de los estados financieros.

En la Comisión elaboradora de los anteriores principios y normas de contabilidad participan representantes de distintas instituciones públicas y privadas, que cuentan entre sus miembros con quienes elaboran, auditan o utilizan los estados financieros de las empresas. Baste citar la pertenencia a dicha Comisión de los representantes de: Asociación Española de Ejecutivos de Finanzas, Banco de España, Colegios de Titulares Mercantiles, Instituto de Analistas de Inversiones, Instituto de Auditores Internos, Instituto de Censores Jurados de Cuentas de España, Instituto de Contabilidad y Auditoría de Cuentas, Intervención General de Administración del Estado y Registro de Economistas Auditores.

Gracias a este esfuerzo de participación de profesionales, y de entidades $y$ organismos que agrupan a éstos, los principios y normas de contabilidad emitidos por AECA han alcanzado hoy día en España un elevado grado de aceptación profesional, no siendo ajeno a ello el hecho de que las distintas corporaciones profesionales de auditores existentes a la fecha en nuestro país hayan reconocido los mismos como generalmente aceptados. El recién creado Instituto de Contabilidad y Auditoría de Cuentas abre un nuevo cauce de respaldo a los anteriores principios y normas, que supondrá sin duda una implantación más 
generalizada de éstas.

Con la incorporación de España en la $\mathrm{CEE}$, nuestras ya viejas leyes de naturaleza mercantil, que tradicionalmente han venido recogiendo los criterios contables con respaldo legal, están siendo objeto de una profunda modificación. En efecto, en estos momentos se encuentra ya en vigor la Ley de Auditoría de Cuentas y en trámite legislativo el Proyecto de Ley sobre "Reforma parcial y adaptación de la legislación mercantil a las Directivas de la CEE en materia de sociedades".

\section{La Ley sobre Auditoría de Cuentas} supone algo más que la mera introducción en nuestro ordenamiento jurídico del contenido de la 8를 Directiva anteriormente comentada, ya que no solamente incluye en su articulado disposiciones sobre los requisitos que han de tener quienes deseen acceder a la profesión de auditor de cuentas, sino que incluye también otras cuestiones de notoria importancia, como por ejemplo son: las empresas obligadas a realizar una auditoría, la dependencia de los auditores de un órgano de la Administración, la posibilidad de que desde la Administración pueda accederse a la documentación y demás papeles de trabajo manejados por los auditores, etc.

La Ley de Reforma parcial de la legislación mercantil en materia de sociedades, supone una modificación del Código de Comercio, la Ley de Sociedades Anónimas y la Ley de Sociedades de Responsabilidad Limitada para adaptar su contenido a lo dispuesto en las Directivas 14, 2a, 3a, 4a, 6! y 7a de derecho societario de la CEE. Entre las anteriores, básicamente son la $4^{\mathrm{a}}$ y la $7^{\mathrm{a}}$ las que contienen disposiciones de natu- raleza contable, según quedó puesto de manifiesto con anterioridad y, en relación con ellas, seguirán nuestros breves comentarios a este respecto.

El título 3 del libro 1 del Código de Comercio relativo a la contabilidad de los comerciantes ha quedado estructurado en el proyecto en varias secciones; una primera que sigue refiriéndose al tema de los libros y otras dedicadas respectivamente a las cuentas anuales y a las cuentas consolidadas. Temas tales como imagen fiel, principios contables básicos, contenido de las cuentas anuales y de las cuentas consolidadas, menciones que obligatoriamente han de realizarse en el anexo o memoria, contenido del informe de gestión, criterios de valoración y auditoría, forman parte de su articulado, recogiendo cuanto a este respecto contienen las Directivas a que anteriormente hicimos referencia. Tan literalmente se han traducido ciertos pasajes de las Directivas que resultan difícilmente comprensibles, inclusive para expertos. Esperamos que el legislador mejore sensiblemente el texto que se le presenta, porque en otro caso su aplicación dará lugar a múltiples problemas interpretativos, fácilmente corregibles de antemano si se atienden las indicaciones de quienes pueden tener una opinión formada sobre el particular ${ }^{(6)}$.

La Ley de Sociedades Anónimas sustituye su antiguo capítulo 6o relativo al balance por un nuevo capítulo $7^{\circ}$ dedicado a las cuentas anuales, en el que se matizan algunas de las cuestiones ante-

(6) AECA viene realizando una importante tarea en este sentido. A su inicial dictamen de 1979 cabe añadir el publicado en junio de 1988, asf como las más recientes notas especfficas enviadas en diciembre de 1988 al Instituto de Contabilidad y Auditorfa de Cuentas. 
riormente tratadas en el Código de Comercio, incluyendo además esquemas sintéticos y más desarrollados para la preparación del balance y de la cuenta de pérdidas y ganancias, reglas de valoración para las principales partidas contenidas en los anteriores estados contables, menciones muy precisas para ser incluidas en la memoria o anexo y en el informe de gestión, obligación de auditar las cuentas anuales anteriores, indicando el proceso para el nombramiento de los auditores, así como el contenido de su informe, forma en que han de darse a la publicidad las anteriores cuentas anuales, informe de gestión e informe de los auditores, y otras variadas disposiciones sobre temas de diversa índole, como por ejemplo son las relativas a las acciones propias o a la distribución de dividendos a cuenta, etc.

A partir de la promulgación de las dos leyes anteriormente citadas, buena parte de las normas contables usualmente aplicadas por los profesionales habrán adquirido rango legal. No debe pensarse que con ésto acaba la tarea de normalización, pues la riqueza y variedad de operaciones que, de día en día, surgen en el mundo de los negocios, exigen tomas de postura por los profesionales, por sus organizaciones, con objeto de reducir a una unicidad, la posible diversidad de criterios que podría surgir y que sin duda surgiría para su tratamiento contable. Por ello, la tarea que en España ha acometido la Asociación Española de Contabilidad y Administración de Empresas (AECA), encuentra si cabe todavía mayor razón de ser después de la promulgación de las anteriores disposiciones, como ocurre en otros países económicamente avanza- dos que desde hace tiempo tienen reconocidos en sus leyes los criterios antedichos, en los que existen asociaciones $u$ organismos que se ocupan de manera permanente de la normalización contable. Tal es el caso de la Orden de expertos contables en Francia, del Accounting Standard Committee en Gran Bretaña o del Financial Accounting Standard Board en Estados Unidos.

Nuestro Plan General de Contabilidad tras las disposiciones anteriores llega por fin a encontrar su normal acomodo, pues es una disposición final del Proyecto de Ley de reforma parcial de la legislación mercantil, la que faculta al Gobierno para la modificación del mismo, con el fin de adaptarle al contenido de las nuevas normas legales, abandonando así la dependencia jurídica de disposiciones fiscales que, en el momento de su nacimiento, le otorgaron la cobertura legal correspondiente.

Como en tantos otros temas, España ha tenido que recorrer, en un período de tiempo mucho más breve que otros países, todo el proceso de regulación contable que lleva a la emisión de normas, tanto en el plano legal como en el profesional. Sin dichas normas las cuentas anuales de nuestras empresas difícilmente podrán llegar a alcanzar el grado de objetividad y de fiabilidad que una sociedad económicamente avanzada necesita. Expresiones tales como imagen fiel y otras de corte similar quedarian en mera retórica si la consecución de tal imagen no puede basarse en unas reglas precisas, en unas normas consagradas por las leyes las más básicas y fruto de la experiencia, y del respaldo de los profesionales las más cambiantes. 


\section{Bibliografias}

\section{El papel de la Teoría Contable}

AMERICAN INSTITUTE OF CERTIFIED PUBLIC ACCOUNTANTS, Objectives of Financial Statements (Trueblood Report). Report of the Study Group on the Objectives of Financial Statements. New York: AICPA, 1973.

ARROW, K.J., Social Choice and Individual Values, Cowles Foundation Monograph. New York: John Wiley, 1963.

R.J. And P. Brown, “An Empirical Evaluation of Accounting Income Numbers”, Journal of Accounting Research 6 (Autumn 1968), pp. 159-178.

BEAVER, W.H., "Financial Ratios as Predictors of Failure", Empirical Research in Accounting: Selected Studies 1966, supplement to Vol, 4 of Journal of Accounting Research (1966), pp. 71-111.

1981.

BENSTON, G.J., Published Corporate Accounting Data and Stock Prices", Empirical Research in Accounting: Selected Studies 1967, supplement to Vol. 5 of Journal of Accounting Research (1967), pp. 1-14 and 22-54.

BLAUG, M., The Methodology of Economics. Cambridge University Press, 1980.

CHAMBERS, R.J., Accounting, Evaluation and Economic Behavior. Englewood Cliffs, N.J.: Prentice-Hall, 1966.

DYCKMAN, T.R., And S.A. Seff, "Two Decades of the Journal of Accounting Research: Journal of Accounting Research 22 (Spring 1984), pp. 225-297.

EDWARDS, E.O., And P.W. Bell, The theory and Measurement of Business Income. Berkeley: University of California Press, 1961.

FRIEDMAN, M., "The Methodology of Positive Economics", Essays in Positive Economics. Chicago: University of Chicago Press, 1953, reimpreso por Chicago: Phoenix Books, 1966.

PASSMORE, J.A., "Can the Social Sciences Be Value-Free?" in H. Feigl and M. Brodbeck, eds., Readings in the Philosophy of Science, pp. 674-676. New York: Appleton-Century-Crofts, 1953, pp. 674-676.

POPPER, K.R., Conjectures and Refutations: The Growth of Scientific Knowledge. London: Routledge \& Kegan Paul, 1963.

New York: Harper Torch Books, 1965. 


\section{Posibilidad y utilidad de la Teoría Positiva de la Contabilidad}

Auerbach, J.S., Enmity and Amity: Law Teachers and Practitioners, 1901)-1922, Perspectives in American History (1971) pp. 551-601.

Bhaskar, R., A Realist Theory of Science (Leeds: Leeds Books, 1975).

Bhaskar, R., The Possibility of Naturalism (Brighton: Harvester, 1979).

Blaug, M., The Methodology of Economics (Cambridge: Cambridge University Press, 1980).

Bledstein, B.J., The Culture of Professionalism (New York: Norton, 1976).

Caldwell, B., Beyond Positivism: Economic Methodology in the Twentieth Century (London: Allen and Unwin, 1982).

Calvert, M.A., The Mechanical Engineer in America, 1830-1900 (Baltimore: John Hopkins University Press, 1967).

Chandler, A.D., The Visible Hand (Cambridge, MA: Harvard University Press, 1977).

Chandler, A.D., Historical Determinants of Management Hierarchies en Van de Ven, A.H. and Joyce, W.F. (eds.) Perspectives on Organization Design and Behaviour, (New York: John Wiley, 1981).

Channell, D.F., The Harmony of Theory and Practice: The Engineering Science of W.J.M. Rankine, Technology and Culture (1982), pp. 39-52.

Christenson, C., The Methodology of Positive Accounting, The Accounting Review (1983) pp. 1-22.

Coats, A.W., Introduction, in Coats A.W. (ed.) Economists in Government (Durham, NC: Duke University Press, 1981).

Coddington, A., Positive Economics, Canadian Journal of Economics (1972) pp. 1-15.

Collins, H., The Seven Sexes: A Study in the Sociology of a Phenomenon or the Replication of Experiments in Physics, Sociology (1975) pp. 205-224.

Collins, H. (ed,), Knowledge and Controversy: Studies of Modern Natural Science, special issue of Social Studies of Science (1981), pp. 1-158.

Daele, W. v.d., The Social Construction of Science en Mendelsohn, E, et al, (eds.) The Social Production of Scientific Knowledge, Sociology of the Sciences Yearbook 1, (Dordrecht: Reidel, 1977).

Daems, H., The Determinants of the Hierarchical Organization of Industry, en Francis A, et al. (eds.) Power, Efficiency and Institutions (London: Heinemann, 1983).

Deane, P., The Scope and Method of Economic Science, Economic Journal'(1983) pp. 1-12.

Dyckman, T.R. \& Zeff, S.A., Two Decades of the Journal of Accounting Research, Journal of Accounting Research (1984) pp. 225-297.

Eichner, A.S., Why Economics is Not Yet a Science, en Eichner, A.S. (ed.) Why Economics is Not Yet a Science (London: Macmillan, 1983).

Farley, J. \& Geison G.L., Science Politics and Spontaneous Generation in Nineteenth Century France: The Pasteur-Pouchet Debate, en Chant, C. and Fauvel, J. (eds.) Darwin to Einstein: Historical Studies on Science and Belief (London: Longman, 1980). 
Fay, Brian, Social Theory and Political Practice (London: Allen and Unwin, 1975).

Feyerabend, Paul K., Problems of Empiricism, en Colodny R. (ed.) Beyond the Edge of Certainty, (Englewood Cliffs, NJ: Prentice-Hall, 1965).

Feyerabend, P.K., How to be a Good Empiricist, en Nidditch, P. (ed.) The Philosophy of Science, (Oxford: Oxford University Press, 1968).

Feyerabend, P.K., Consolidations for the Specialist, en Lakatos, I. and Musgrave, A. (eds.) Criticism and the Growth of Knowledge (Cambridge: Cambridge University Press, 1970).

Feyerabend, P.K. (ed.), Against Method (London: New Left Books, 1975).

Feyerabend, P.K., The Methodology of Scientific Research Programmes, en Feyerabend, P.K. (ed.) Problems of Empiricism, Philosophical Papers, Vol. 2 (Cambridge: Cambridge University Press, 1981).

Findlay, M.C. \& Williams, E.E., A Positivist Evaluation of the new Finance, Financial Management (1980) pp. 7-17.

Findlay, M.C. \& Williams, E.E., A Post-Keynesian View of Modern Financial Economics: In Search of Alternative Paradigms, Journal of Business Finance and Accounting (1985) pp. 1-18.

Freidson, E., Profession of Medicine (New York: Dodd, Mead, 1970).

Freidson, E., Professional Powers (University of Chicago Press, 1986).

Gordon, R.A. and Howell, J.E., Higher Education for Business (New York: Columbia University Press, 1959).

Hall, J.R., An Issue-oriented History of T.I.M.S., Interfaces (1983) pp. 9-19.

Harré, R., The Principles of Scientific Thinking (London: Macmillan, 1970).

Harré, R., Social Being (Oxford: Blackwells, 1979).

Hopwood, A., On Trying to Study Accounting in the Contexts in Which it Operates, Accounting Organizations and Society (1983) pp. 287-305.

Hopwood, A. \& Bromwich, M., Accounting Research in the United Kingdom, en Hopwood, A. and Schreuder, H. (eds.) European Contributions to Accounting Research (Amsterdam: Free University Press, 1984).

Hutchison, T., On the History and Philosophy of Science and Economics, en Latsis, S. (ed.) Method and Appraisal in Economics (Cambridge University Press, 1976).

Hutchison, T., Our Methodological Crisis, en Wiles, P. and Routh, G. (eds.) Economics in Disarray (Oxford: Blackwells, 1984).

Jensen, M.C., Capital Markets: Theory and Evidence, Bell Journal of Economics and Management (1972) pp. 357-398.

Kaplan, R.S., The Role for Empirical Research in Management Accounting, Accounting, Organizations and Society (1986) pp. 429-452.

Karpik, L., Organizations, Institutions and History, en Karpik, L. (ed.) Organization and Environment (London: Sage, 1978).

Kay, N.. The Emergent Firm (London: Macmillan, 1984). 
Kevles, D., The Physicist (New York: Alfred A. Knopf, 1977).

Knorr, K., Krohn, R. and Whitley, R. (eds.), The Social Process of Scientific Investigation Sociology of the Sciences Yearbook 4 (Dordrecht: Reidel, 1981).

Lakatos, I., Falsification and the Methodology of Scientific Research Programmes, en Lakatos, I. and Musgrave, A. (eds.) Criticism and the Growth of Knowledge (Cambridge University Press, 1970).

Lakatos, I., History of Science and its Rational Reconstructions, en Buck, R. and Cohen, R. (eds.) Boston Studies in the Philosophy of Science 8 (Dordrecht: Reidel, 1971).

Larson, M.S., The Rise of Professionalism (University of California Press, 1977).

Levy, R.M., The Professionalization of American Architects and Civil Engineers, Tesis de PhD no publicada, University of California at Berkeley (1980).

Locke, R.R., The End of the Practical Man: Entrepreneurship and Higher Education in Germany, France and Great Britain, 1880-1940 (Greenwich, CT: JAI Press, 1984).

Lowe, E.A., Puxty, A.G. \& Laughlin, R.C., Simple Theories for Complex Processes: Accounting Policy and the Market for Myopia, Journal of Accounting and Public Policy (1983) pp. 19-42.

Macdonald, K.M., Social Closure and Occupational Registration, Sociology (1985) pp. 541-556.

Mattessich, R., Methodological Preconditions and Problems of a General Theory of Accounting, Accounting Review (1972) pp. 469-487.

Merchant, C., The Death of Nature (New York: Harper and Row, 1980).

Musgrave, A., Unreal Assumptions in Economic Theory: The F-Twist Untwisted, Kyklos (1981), pp. 377-387.

Peasnell, K.V. \& Williams, D.J., Ersatz Academics and Scholar-Saints: the Supply of Financial Accounting Research, Abacus (1986) pp. 121-135.

Pierson, F.C., et al., The Education of American Businessmen (New York: McGraw-Hill, 1959).

Popper, K., Conjectures and Refutations (London: Routledge and Kegan Paul, 1963).

Popper, Karl, The Logic of Scientific Discovery (London: Hutchinson, 1968).

Pinch, Trevor, Confronting Nature (Dordrecht: Reidel, 1986).

Prigogine, I. \& Stengers, I., Order out of Chaos (London: Heinemann, 1984).

Reich, L.S., The Making of American Industrial Research (Cambridge University Press, 1985).

Ross, S., The Current Status of the Capital Asset Pricing Model, Journal of Finance (1978) pp. 885-901.

Ryan, R.J., Capital Market Theory - A Case Study in Methodological Conflict, Journal of Business Finance and Accounting (1982) pp. 443-458.

Schreuder, H., Positively Normative (Accounting) Theories, en Hopwood, A. and Schreuder, H. (eds.) European Contributions to Accounting Research (Amsterdam: Free University Press, 1984).

Seely, B.E., The Scientific Mystique in Engineering, Highway research at the Bureau of Public Roads 1918-1940, Technology and Culture (1984) pp. 798-831.

Stockman, N., Antipositivist Theories of the Sciences (Dordrecht: Reidel, 1983). 
Tinker, T., Paper Prophets (New York: Praeger, 1985).

Thomas, D., Naturalism and Social Science (Cambridge University Press, 1979).

Thomas, T.T., Towards a Value-neutral Positive Science of Accounting, Journal of Business Finance and Accounting (1981) pp. 549-572.

Tomkins, C. \& Groves, R., The Everyday Accountant and Researching his Reality, Accounting, Organizations and Society (1983) pp. 361-374.

Turner, S.P., Sociological Explanation as Translation (Cambridge University Press, 1980).

Watts, R.L. \& Zimmerman, J.L., Positive Accounting Theory (Englewood Cliffs, NJ: Prentice-Hall, 1986).

Whitley, R., The Fragmented State of Management Studies, Reasons and Consequences, Journal of Management Studies (1984) pp. 331-348.

Whitley, R., The Transformation of Business Finance into Financial Economics: The Roles of Academic Expansion and Changes in U.S. Capital Markets, Accounting Organizations and Society (1986) pp. 171-192.

Weber, M., The Methodology of the Social Sciences (New York: Free Press, 1949).

Wiebe, R.H., The Search for Order, 1877-1920 (New York: Hill and Wang, 1967).

Williamson, Oliver E., Markets and Hierarchies (New York: Free Press, 1975).

Wynne, Brian, C.G., Barkla and the J. Phenomenon, Social Studies of Science (1976) pp. 307-347.

\section{Políticas y normas contables}

\section{en una muestra de sociedades anónimas del Valle del Cauca}

ACUÑA M. Henao H. Prácticas Contables en el Valle del Cauca. Revista Cuadernos de Administración, No. 11 Univalle 1985, pp. 39-53.

BARONA B., Burbano J. y Sinisterra G. Programa de Investigación en Información Financiera Pública, Mimeo. Univalle 1987.

BALLESTEROS Enrique. Teoría y Estructura de la Nueva Contabilidad. Alianza Universidad Textos. Madrid 1979.

BALL Ray y FOSTER George. Corporate Financial Reporting. A Methodological Review of Empirical Research. Vol 20. USA 1982.

BELKAOUI Ahmed. Accounting Theory Editorial H.B.J. Inc. New York 1981.

BEAVER William. Financial Reporting and Accounting Revolution. Editorial PHI. New Jersey 1981.

BLANCO L. Yanel. Las normas de Contabilidad en Colombia. Edit. Roesga 1987.

BRIONES Guillermo. La Formulación de Problemas de Investigación Social. Ediciones Uniandes. Bogotá 1981.

BRUNS y De Coster D. La Contabilidad y el Comportamiento Humano. Editorial Trillas: México 1975. 
CARDONA John. Estructura Básica de la Contabilidad para Colombia. Revista No. 14 Contaduría Universidad de Antioquia. Medellín 1989, pp. 43-111.

CARDONA John. La Revelación Contable - Un Estudio Exploratorio. Revista No. 8 Universidad de Antioquia. Medellín 1986, pp 77-111.

Cámara de Comencio de Cali. Boletín Estadístico sobre Inversión Privada en el Valle del Cauca. Cali 1986.

Cámara de Comercio de Bogotá. Decreto 2160 de 1986.

CAÑIBANO Leandro. Teoría Actual de la Contabilidad. Biblioteca de Ciencias Empresariales Ediciones ICE. Madrid 1979.

GEORGE Claude. Historia del Pensamiento Administrativo Editorial PHI. México 1984.

HANAN Mack. Incremento de utilidades. Cómo acelerarlo mediante la aplicación de estrategias empresariales. Editorial Norma. Bogotá 1982.

HENDRICKSEN Eldon. Teoría de la Contabilidad. Edit. Uteha. México 1981.

HORNGREN Charles. Contabilidad Administrativa Introducción. Edit PHI. Carvajal. Cali 1983.

KAST. F. y ROSENZWEIG J. Administración en las Organizaciones. Un enfoque de Sistemas. Edit. Mc Graw Hill. México 1980.

MATTESICH Richard. Un examen científico aplicado para una estructura metodológica. Revista Teuken No. 3. C. Rivadavia. Argentina 1988.

MCNICHOLS Thomas. Política Empresarial. Edit. Mc Graw Hill. Bogotá 1981.

MLLER Martin. Guía de Principios de Contabilidad generalmente aceptados. Editorial H.B.J. New York 1982.

MORRISSEY Leonard. Teoría Contable de la Información Financiera. Editorial Trillas. México 1977.

LEE Thomas. Developments in Company Financial Reporting a History and an Introduction. Philip Alan Publishers Limited. Oxford 1981.

LIBBY Robert. Accounting and Human Information Processing: Theory and Aplications. Edit. Prentice Hall Inc. Englewood Cliffs. New Jersey 1981.

PARDINAS Felipe. Metodología y Técnicas de Investigación en Ciencias Sociales. Editorial "Siglo XXI Editores". México 1983.

RODRIGUEZ Mario. La información contable y su exposición. Administración de Empresas. Tomo II.

SHIM J. y SIEGEL J. Contabilidad Administrativa - Ser ie Schaum. Editorial Mc Graw Hill. Bogotá 1986.

SIMKIN M. y MOSCOVE Stephen. Accounting Information Systems Concepts and practice for effective decision. Makin Edit. John Wiley y Sons Inc. New York 1979.

TUA Pereda Jorge. Principios y Normas de Contabilidad, impreso en la Fábrica Nacional de Moneda y Timbre. Madrid 1983.

UDUAL. IV Conferencia de Facultades y Escuelas de Contaduría Pública de América Latina - III Congreso Latinoamericano de Investigación Contable. Universidad Central. Bogotá 1986. 
VASQUEZ José. Contabilidad Moderna. Edit. Bedout. Medellín 1947.

VLAEMMINCK Joseph. Historia y doctrinas contables. Editorial Index. Madrid 1961.

WATTZ y ZIMMERMAN. Positive Accounting Theory. Editorial PHI. New Jersey 1986.

ZEFF Stephen. Evolución de la Teoría Contable. La investigación empírica. Revista Contaduría Universidad de Antioquia. No. 6 Marzo de 1985, pp 25-53. 